\title{
Infant psychiatric disorders
}

\author{
Margarete I. Bolten
}

Published online: 11 December 2012

(c) Springer-Verlag Berlin Heidelberg 2012

\begin{abstract}
Infant mental health problems include difficulties to regulate emotions or attention, crying, sleeping or feeding problems as well as aggressive behavior. Early identifications of these problems help to change developmental trajectories and improve developmental outcomes. Psychiatric assessment and classification have to take into account the rapid processes of development as well as the inseparable linkage between symptoms of the infant, psychosocial risks in the family environment, and parent-child relations. The proposed DSM-5 classification system presents a systematic description of mental health disorders which are relevant for infant psychiatry. However, the proposal has provided rather limited attention to developmental differences and parent-infant relations. Therefore, additional classification systems, like the Zero-to-Three (DC: 0-3R), are strongly recommended. In terms of assessment and in accordance with the guidelines of the American Academy of Child and Adolescent Psychiatry, infant psychiatrists have to consider the close relation between somatic and mental health and the interplay between behaviors of the caregiver and the infant. Therefore, the assessment has to be multidisciplinary and relationship based. A standard assessment in infancy includes a clinical interview, behavior observations, caregiver questionnaires, and a pediatric screening. All assessments should pay attention to motor, cognitive, language, and social-emotional development. Because infant development is embedded
\end{abstract}

M. I. Bolten $(\bowtie)$

Department of Developmental Psychopathology, Child and Adolescents Psychiatric Clinic, University of Basel, Schanzenstrasse 13, 4056 Basel, Switzerland e-mail: margarete.bolten@upkbs.ch in the family context, socio-economic factors, parents' mental problems, including drug abuse, domestic violence, and trauma history should be assessed. The treatment has to be oriented toward symptoms and development and has to address underlying medical conditions. The focus should be on parent-child interactions. Evidence-based interventions are based on attachment theory, use social-learning perspectives, and behavioral approaches.

Keywords Infant - Toddler · Feeding disorder - Sleeping disorder - Regulatory problems - Emotion - Cognition . Regulation - Interdisciplinary assessment - DC: 0-3R . RDC-PA

\section{Introduction}

While the public tends to view infancy as a carefree period, very young children already experience significant mental health problems. Since the last three decades, there is a growing recognition that very young children experience impairing mental health problems of clinical relevance. For instance, in the study by Sadeh et al. [1], $23 \%$ of the parents reported sleeping problems of moderate and $2 \%$ of serious impact. Eating problems are also very common in infancy. Prevalence numbers range from 20 to $25 \%$ in the normal population [2-4] and from 40 to $80 \%$ in children with disabilities. Using the Wessel criteria [5], prevalence rates for excessive crying vary as $1.5 \%$ in The Netherlands [6], $9.2 \%$ in Denmark [7], and $16.3 \%$ in Germany [8].

Behavioral problems in infancy can impact the child's development. A quantitative meta-analysis by Hemmi et al. [9] showed that infants with persistent crying, feeding, and 
sleeping difficulties are far more likely to develop significant behavioral problems. Furthermore, infants with behavioral problems are more likely to have impaired parent-child relationships [10]. Therefore, it is mandatory to identify and treat these problems early to improve developmental outcomes and promote positive parentchild relationships. However, our understanding of the epidemiology, assessment, and treatment of mental health problems in infancy lags far behind our knowledge about mental health problems in older children and adolescents.

Psychiatric classification, assessment, and treatment of disorders in infancy have to take into account the rapid motor and psychological development as well as the parent-infant relationship during this early phase of life. Developmentally, sensitive norms have to be used and transient problems have to be distinguished from persistent problems as well as normative variation from clinically significant syndromes.

\section{DSM-5 classification of infant mental disorders}

The DSM-5 classification system presents a description of mental disorders relevant to infant psychiatry. However, the application of the DSM-5 criteria to very young children is challenging because the criteria have been developed with limited awareness of developmental differences in the presentation of psychiatric disorders and the interdependence between the child and his or her caregivers. Nevertheless, the use of these DSM-5 criteria will become important because of their connection to the rest of child and adolescent psychiatry.

Although there is quite a variety of behavior problems in children under the age of five, only the most common mental disorders in infants and toddlers, namely, irritability and crying problems, feeding disorders, and sleeping disorders will be outlined here. In the DSM-5, only feeding and sleeping disorders are listed. For irritability and crying problems, alternative classification systems have to be used.

\section{00 Insomnia Disorder}

The DSM-5 Sleep-Wake Disorders Work Group recommends seven changes in the "Insomnia Disorder" diagnostic criteria for consideration in the revised classification. Table 1 outlines the proposed changes for the diagnosis of "Insomnia Disorder".

It is proposed to exchange the DSM-IV categories "Primary Insomnia" and "Insomnia related to another mental/medical disorder" by "Insomnia Disorder". Clinically relevant comorbid conditions should be specified separately. The minimum frequency criterion (i.e., 3 nights per week) for the sleep disturbance and the raised minimum duration threshold from 1 to 3 months serves a clear definition of the disorder. Specific examples for daytime impairments of the child caused by the Insomnia Disorder (e.g., irritability, dysphoria) or the examples for the

Table 1 Proposed DSM-5 revision for Insomnia Disorder (updated 1 May 2012)

A. The predominant complaint is dissatisfaction with sleep quantity or quality. In children or the elderly, the complaint may be made by a caregiver or family member

B. Report of one or more of the following symptoms:

1. Difficulty initiating sleep. In children, this may be manifested as difficulty initiating sleep without caregiver intervention

2. Difficulty maintaining sleep, characterized by frequent awakenings or problems returning to sleep after awakenings. In children this may be manifested as difficulty returning to sleep without caregiver intervention

3. Early-morning awakening with inability to return to sleep

4. Non-restorative sleep

5. In children, prolonged resistance to going to bed and/or bedtime struggles

C. The sleep complaint is accompanied by significant distress or impairment in daytime functioning as indicated by the report of at least one of the following:

1. Fatigue or low energy

2. Cognitive impairment (e.g., attention, concentration, memory)

3. Mood disturbance (e.g., irritability, dysphoria)

4. Behavioral problems (e.g., hyperactivity, impulsivity, aggression)

5. Impaired occupational or academic functioning

6. Impaired interpersonal/social functioning

7. Negative impact on caregiver or family functioning (e.g., fatigue, sleepiness)

D. The sleep difficulty occurs at least three nights per week

E. The sleep difficulty is present for at least 3 months

F. The sleep difficulty occurs despite adequate opportunity for sleep 
Table 2 Proposed DSM-5 category of avoidant/restrictive food intake disorder (updated May 14, 2012

A. Eating or feeding disturbance (including but not limited to apparent lack of interest in eating or food; avoidance based on the sensory characteristics of food; or concern about aversive consequences of eating) as manifested by persistent failure to meet appropriate nutritional and/or energy needs associated with one or more of the following:

1. Significant weight loss (or failure to gain weight or faltering growth in children)

2. Significant nutritional deficiency

3. Dependence on enteral feeding or nutritional supplements

4. Marked interference with psychosocial functioning

B. There is no evidence that lack of available food or an associated culturally sanctioned practice is sufficient to account for the disorder

C. The eating disturbance does not occur exclusively during the course of Anorexia Nervosa or Bulimia Nervosa, and there is no evidence of a disturbance in the way in which one's body weight or shape is experienced

D. The eating disturbance is not better accounted for by a concurrent medical condition or another mental disorder. When occurring in the context of another condition or disorder, the severity of the eating disturbance exceeds that routinely associated with the condition or disorder and warrants additional clinical attention

negative impact on caregiver or family functioning (e.g., fatigue, sleepiness) are very suitable for clinical practice.

\section{K 02 Avoidant/Restrictive Food Intake Disorder}

The current DSM-IV criteria for "Feeding Disorder of Infancy or Early Childhood" will be replaced by some proposed changes for a new category of "Avoidant/ Restrictive Food Intake Disorder" as outlined in Table 2.

The DSM-5 Eating Disorders working group recommended to change the name of the current category of "Feeding Disorder of Infancy or Early Childhood" into "Avoidant/Restrictive Food Intake Disorder" to reflect the fact that feeding disorders are present in children for longer periods rather than infancy and early childhood only [12]. Because the DSM-IV criteria for Feeding Disorders have been criticized for failing to identify many very young children with feeding difficulties, changes in the DSM criteria have become necessary. The main characteristic of this disorder is the restriction or avoidance of food intake. The revision will include a description of three main subtypes which have been identified in the literature [12]:

- children who do not eat enough respectively show little interest in feeding,

- children who accept only a limited diet in relation to specific sensory features,

- children in whom food refusal is related to aversive experience.

The DSM-5 will also include a dimensional assessment for severity of the feeding disorders. The severity can be classified from 0 (no symptoms of the disorder) to 4 (diagnostic criteria are fully met and the disorder is severe). Nevertheless, the dimensional proposals are problematic because they have been designed largely ad hoc without substantial empirical evidence.

\section{Alternative Diagnostic Classifications in Early Childhood}

Because the DSM-5 classification will not cover all needs of the infant mental health field, supplementary resources for the classification of mental health problems in early childhood are needed. For instance, the Diagnostic Classification of Mental Health and Developmental Disorders of Infancy and Early Childhood Revised (DC: 0-3R) [14] can be used in clinical practice. For preschoolers, the modified DSM-IV, the Research Diagnostic Criteria-Preschool Age (RDC-PA) [13], may also be suitable. Both the classification systems allow the consistent application of a developmentally sensitive nosology for children up to the age of 5 years.

\section{Guidelines for assessment}

Following the guidelines by the American Academy of Child and Adolescent Psychiatry (AACAP) [15], clinical assessment in infancy has to consider the close relation of somatic and mental health and the interplay between caregivers and infant's behavior. Parents are most important in the therapeutic team. Consequently, infant psychiatrists have to establish a strong work-alliance with the family. The family background including socio-economic factors, parent's mental health problems, domestic violence, and trauma history should be evaluated. The assessment must be multidisciplinary and relationship based. The assessment process has to pay attention to motor, cognitive, language, and social-emotional development and has to consist of four main parts: (a) assessment of the severity of the disorder, (b) assessment of developmental aspects of the disorder, (c) assessment of sustaining factors, and (d) diagnosis by exclusion.

Since infants do not have the same cognitive and emotional competencies and cannot communicate verbally like 
older children or adolescents, infant psychiatrists have to use different approaches. The most important diagnostic tools in infant psychiatry are structured and unstructured video-based behavior observations of the infant and the parent-infant-dyad interaction. A brief separation and reunion may activate the attachment system and provides information, how the infant uses the caregiver as a secure base and how caregiver expresses warmth, sensitivity, affective involvement, and reaction to noncompliance. Besides observations, useful assessment methods are interviews, caregiver questionnaires, and protocols as well as pediatric screenings.

\section{Guidelines for treatment}

\section{General approach}

In infant psychiatry, treatments should be symptom and developmental orientated and should address underlying medical conditions and parent-child interactions. Very often, persistent behavioral problems of the child result in a strong burden to the parents and may have a negative impact on the parent-child relationship [10, 16, 17]. Infants evoke numerous thoughts and feelings in their parents, which may intimidate the infant's development or a positive parent-child relationship. Consequently, a reduction of positive reciprocity, limited responsiveness, and sensitivity of the parents may evolve [18]. Therefore, the main goal of the treatments in infancy is to support parental sensitivity and positive parent-infant interactions. One potential approach may be infant-parent psychotherapy (IPP) [11, 19], an attachment-based psychotherapy to promote healthy development through dyadic play, reflective guidance, and linking parent's caregiving experiences to the interactions with their own child. IPP offers the possibility of thoughtful exploration about parenthood, parent-child relationship, and infant's needs. Since parental perceptions and representations of their infant often may be observed best in the presence of the infant, the therapist offers the opportunity to detect, understand, and express such thoughts and feelings to the parents in the presence of their child. There is an evidence that this procedure results in positive changes in parent perceptions of the infant, self-perception, and maternal sensitivity [20].

In general, an out-patient treatment is sufficient for the treatment of crying problems and sleeping disorders in infancy. However, not all feeding disorders can be treated by this approach. Hospitalization may be required to accomplish structured mealtimes and stricter limit setting, by the parents to facilitate internal regulation of eating. Especially when regular mealtimes with periods of food abstinence are scheduled, close monitoring of the child and support of the parents are required so that inpatient treatment may become mandatory. Out-patient treatment should also be avoided in the presence of an increased risk of neglect or abuse, several comorbid disorders (crying, sleeping, or feeding), significant impairment of the infant's development, and mental disorders in the parents, which may be associated with a substantial perceptual distortion of infant signals or lack of parental skills. In these instances, intensive in-patient mother child therapy should be considered. In cases of parental mental disorders or marital conflicts, additional individual, family, or couples therapy may be indicated.

There is no evidence regarding the efficacy and safety of psychopharmacologic treatment in infancy. Medication has to be used with caution in this age group and should be considered only after a trial of appropriate psychotherapy has failed.

\section{Treatment of irritability and crying problems}

The treatment of irritability and crying problems should help the parents to recognize and use behavior signals of their child to support the infant's self-regulation. In particular, the following therapeutic elements are useful: (a) psycho-education and developmental consultation, (b) relief of the parents, (c) reduction of overstimulation and structuring of the day, (d) break-through of the negative interaction circles, and (e) relapse prevention.

Currently, there are only a few randomized controlled trials on the efficacy of different therapeutic approaches for excessive crying. There is evidence that interventions based on attachment theory [21-23] or the use sociallearning and behavioral approaches work [24-27]. However, the evaluation of the effectiveness of interventions for excessive crying may be rather unsatisfactory because of the spontaneous remission of the problem at the age of 3 months. Wolke et al. [28] compared the effect of a complex behavioral intervention for excessive crying with an empathy group (talking through the problem) and with a control group receiving no intervention. The reduction of crying by the complex behavioral treatment was significantly bigger (by $51 \%$ ) than by other the two groups (37\% in the empathy group and $35 \%$ in the control group).

Treatment of sleep disorders

Cognitive-behavioral therapy (CBT) is an evidence-based intervention for sleep problems [20]. CBT uses strategies for enhancing overall parenting effectiveness as well as improving sleep problems in terms of the following approaches: 
1. Preventive parent education: Parents are informed how sleep-behavior develops in infancy, how much infants sleep in general and which factors may impact the infant's sleep. Furthermore, caregivers are instructed to disassociate feeding from sleep-wake transitions to establish a stable sleep pattern early in the infant's life.

2. Extinction technique (systematic ignoring): This technique involves establishing a regular bedtime routine, then placing the child awake into the bed and to ignore all protests until an established time in the next morning.

3. Graduated extinction (Ferber technique): Because many parents may perceive pure extinction as too cruel, modifications to the procedure have been designed. The protocol involves an increasing reduction of parental attention to inappropriate bedtime behaviors. This may allow the child to fall asleep without parental assistance.

4. Stimulus control technique (Positive routine): This approach involves temporarily moving the bedtime later into the evening to get closer to the child's natural sleep onset time. Parents should introduce a positive and enjoyable pre-bedtime routine that teaches the child to engage in relaxing activities. The pleasurable activities are stopped immediately if the child protests or shows tantrums. Once the behavioral chain is well established and the child is falling asleep quickly, the bedtime is systematically moved earlier into the evening until reaching the goal of a pre-established bedtime.

5. Scheduled awakenings: Parents wake the child approximately $15 \mathrm{~min}$ before his or her typical nightly awakening times. The scheduled awakenings then are gradually stopped or faded out. Scheduled awakenings systematically increase the length of children's sleep periods while eliminating spontaneous awaking.

6. Treatment for sleep-related fears and anxiety: These techniques are more important for preschool children and include relaxation training, guided imagery, selftalk, positive reinforcement for increasingly successful efforts, systematic desensitization, and gradual exposure to a child-determined hierarchy of sleep-related fears or anxiety.

Sometimes, the implementation of these rather directive approaches fail because of fears, aggression, or sometimes even negative memories of the parents. In these instances, IPP [11] might be indicated.

\section{Treatment of feeding disorders}

Besides the basic principles for treatments in infancy, some special features have to be considered in the case of feeding disorders. Therapy modules include breastfeeding advice in newborns or young infants. Inadequate perception of the infant's signals, incorrect holding, too frequent or too rare breastfeeding, too large and too small amounts of feeding, and coordination problems of feeding and breathing may be subject of such a nursing advice. In addition, general feeding and eating rules might be of great help [29]. The infant should be fed only at fixed mealtimes. To increase the infant's appetite and hunger at mealtimes, no other foods and drinks except water should be offered between meals. The duration of each meal should not last for more than $30 \mathrm{~min}$. Mealtimes should take place in a neutral atmosphere without distraction and parents should not feed with coercion. Food should never be offered as a reward or gift. Small portions of solid food should be offered first. The child gets fluids later so that the appetite will not be suppressed. The autonomy of the infant should be supported by the parents. Negative mealtime behaviors (tantrums, playing or throwing with food) should be ignored whereas positive behavior should be praised by the parents. In case of swallowing and chewing difficulties, a training of oral sensitivity and oral motor skills by specialized therapists (e.g., speech therapist) can be very helpful.

\section{Summary}

Both in clinical practice and in research, infant psychiatry requires a true integration of biological, psychological, and social intervention strategies with collaboration across disciplines. Infant psychiatrists can intervene early to promote current and future healthy development and relationships, and can treat a wide range of psychopathology in infants. The growth and development of this specialty, like early childhood development itself, is rapid and holds extraordinary potential to promote family mental health and treat psychopathology.

Conflict of interest The author states that there are no conflicts of interest. This article is part of the supplement "The Future of Child and Adolescent Psychiatry and Psychology: The Impact of DSM 5 and of Guidelines for Assessment and Treatment". This supplement was not sponsored by outside commercial interests.

\section{References}

1. Mindell JA et al (2009) Co-sleeping, parental presence, and sleep in young children: a cross-cultural perspective. Sleep 32:A81-A82

2. McDermott BM et al (2008) Preschool children perceived by mothers as irregular eaters: physical and psychosocial predictors from a birth cohort study. J Dev Behav Pediatr 29(3):197-205

3. Wright CM et al (2007) How do toddler eating problems relate to their eating behavior, food preferences, and growth? Pediatrics 120(4):e1069-e1075

4. Chatoor I (2009) Diagnosis and treatment of feeding disorders in infants, toddlers and young children. Zero to Three Press, Washington DC 
5. Wessel MA et al (1954) Paroxysmal fussing in infancy, sometimes called "colic". Pediatrics 14:421-435

6. Reijneveld SA, Brugman E, Hirasing RA (2001) Excessive infant crying: the impact of varying definitions. Pediatrics 108(4): 893-897

7. Alvarez M (2004) Caregiving and early infant crying in a danish community. J Dev Behav Pediatr 25(2):91-98

8. von Kries R, Kalies H, Papousek M (2006) Excessive crying beyond 3 months may herald other features of multiple regulatory problems. Arch Pediatr Adolesc Med 160(5):508-511

9. Hemmi MH, Wolke D, Schneider S (2011) Associations between problems with crying, sleeping and/or feeding in infancy and long-term behavioural outcomes in childhood: a meta-analysis. Arch Dis Child 96(7):622-629

10. Papousek M, von Hofacker N (1998) Persistent crying in early infancy: a non-trivial condition of risk for the developing motherinfant relationship. Child Care Health Dev 24(5):395-424

11. Lieberman AF (1992) Infant parent psychotherapy with toddlers. Dev Psychopathol 4(4):559-574

12. Bryant-Waugh $\mathrm{R}$ et al (2010) Feeding and eating disorders in childhood. Int J Eat Disord 43(2):98-111

13. Scheeringa $M$ et al (2003) Research diagnostic criteria for infants and preschool children: the process and empirical support. J Am Acad Child Adolesc Psychiatry 42(12):1504-1512

14. Zero-to-Three (2005) Zero to Three DC: 0-3R diagnostic classication of mental health and developmental disorders of infancy and early childhood, Revised Edition. Zero to Three Press, Washington DC

15. Thomas JM (1998) Summary of the practice parameters for the psychiatric assessment of infants and toddlers (0-36 months). American Academy of Child and Adolescent Psychiatry. J Am Acad Child Adolesc Psychiatry 37(1):127-132

16. Meijer AM, van den Wittenboer GL (2007) Contribution of infants' sleep and crying to marital relationship of first-time parent couples in the 1st year after childbirth. J Fam Psychol 21(1):49-57

17. Wake $M$ et al (2006) Prevalence, stability, and outcomes of cryfuss and sleep problems in the first 2 years of life: prospective community-based study. Pediatrics 117(3):836-842
18. Papoušek M, Papoušek H (1990) Exzessive infant crying and intuitive parental care. Buffering support and its failures in parent-infant interaction. Early Child Dev Care 65:117-126

19. Jordan B (2012) Therapeutic play within infant-parent psychotherapy and the treatment of infant feeding disorders. Infant Mental Health J 33(3):307-313

20. Mindell JA et al (2006) Behavioral treatment of bedtime problems and night wakings in infants and young children-An American Academy of Sleep Medicine review. Sleep 29(10): $1263-1276$

21. Cramer B et al (1990) Outcome evaluation in brief mother-infant psychotherapy: a preliminary report. Infant Mental Health $\mathbf{J}$ 11:278-300

22. Robert-Tissot $C$ et al (1996) Outcome evaluation in brief motherinfant psychotherapies: report on 75 cases. Infant Mental Health J 17(2):97-114

23. Salomonsson B, Sandell R (2011) A randomized controlled trial of mother-infant psychoanalytic treatment: II. Predictive and moderating influences of qualitative patient factors. Infant Mental Health J 32(3):377-404

24. Wolke D (1993) The treatment of problem crying behavior. St James Roberts I, Harris Jr GS, Messer D (Eds) In: Infant crying, feeding and sleeping: development, problems and treatments. Harvester, New York

25. McKenzie S (1991) Troublesome crying in infants: effect of advice to reduce stimulation. Arch Dis Child 66(12):1416-1420

26. Keefe MR et al (2006) Effectiveness of an intervention for colic. Clin Pediatr (Phila) 45(2):123-133

27. Murray L et al (2003) Controlled trial of the short- and long-term effect of psychological treatment of post-partum depression: 2 . Impact on the mother-child relationship and child outcome. Br J Psychiatry 182:420-427

28. Wolke D, Gray P, Meyer R (1994) Excessive infant crying: a controlled study of mothers helping mothers. Pediatrics 94:322-332

29. Bernard-Bonnin AC (2006) Feeding problems of infants and toddlers. Can Fam Physician 52:1247-1251 